\title{
SUPPRESSION OF TRANSVERSE BUNCH INSTABILITIES BY ASYMMETRIES IN THE CHAMBER GEOMETRY*
}

\author{
A. Burov, FNAL, Batavia, IL and V. Danilov, ORNL, Oak Ridge, TN
}

\section{Abstract}

The wake forces produced by a beam bunch can be reduced by making the vacuum chamber cross-section axially asymmetric. Furthermore, the asymmetry results in a betatron tune shift for particles in the tail of the bunch. As a result, transverse instabilities of the bunch should be significantly suppressed for an asymmetric vacuum chamber.

\section{INTRODUCTION}

The net effect of wake fields is determined by integrating the force over a structure period of the vacuum chamber $L$. The integrated transverse force $\mathbf{F}$ caused by a slight offset $\mathbf{r}_{\mathbf{0}}$ of the leading particle from the chamber axis is conventionally expressed in terms of the wake function [2]:

$$
\int_{L} \mathbf{F} d s=-q^{2} \mathbf{r}_{\mathbf{0}} W(z)
$$

where $q$ is the particle's charge and $z$ is the distance between head and tail particles.

However, the linear approximation for the wake force (1) contains generally an additional term, proportional to the tail offset $\mathbf{r}$ [3], which vanishes for round chambers. It means that, for round chambers, all the particles in the bunch are in resonance with each other. For axially asymmetric structures, however, the wake fields not only drive the oscillations of the tail particles but also detune them from the resonance with the driving force. Similar electrodynamic properties of external RF fields in asymmetric structures were used in Ref. [4], where it was proposed to utilize simultaneous accelerating and focusing to provide the acceleration and BNS damping [5] in linacs.

The importance of the betatron tune spread along a bunch in a storage ring was shown in Ref. [6]. It was demonstrated that this spread, introduced by means of an RF quadrupole, has a stabilizing role for the transverse bunch oscillations. It is natural to suppose that the tune spread produced by the wake fields is a stabilizing factor as well. If so, the detuning part of the wake may increase the thresholds of bunch transverse instabilities.

\section{DRIVING AND DETUNING WAKES}

The transverse wake forces are regular functions of the transverse offsets of the leading and trailing particles, $\mathbf{r}_{\mathbf{0}}$ and $\mathbf{r}$ and can be expanded in terms of these offsets [3]. Assuming for simplicity mirror symmetry for at least one transverse axis and neglecting the nonlinear terms, the

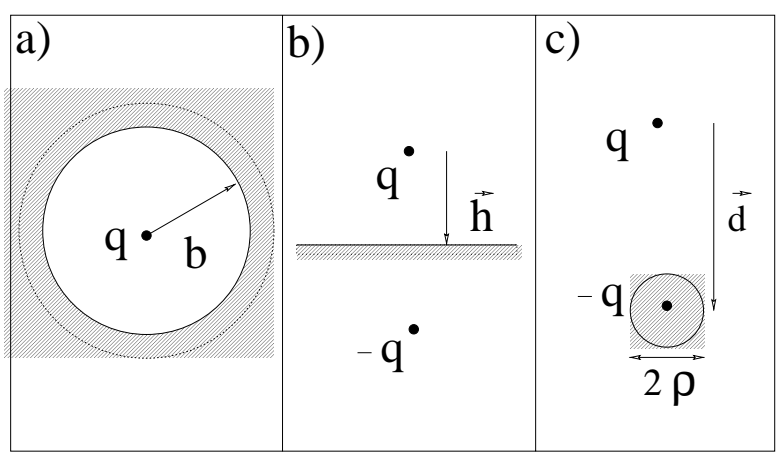

Figure 1: Three types of beam surroundings.

forces can be presented as follows:

$$
\begin{aligned}
& \int_{L} F_{x} d s=-q^{2} x_{0} W_{x}(z)+q^{2} x D(z) \\
& \int_{L} F_{y} d s=-q^{2} y_{0} W_{y}(z)-q^{2} y D(z),
\end{aligned}
$$

where insignificant constant terms are omitted. The first terms on the right hand sides describe the forces caused by the offsets of the leading particle; the functions $W(z)$ can be referred to as the driving wake functions. The second terms are responsible for the tune shifts of the tail particle; the function $D(z)$ can be called the detuning wake function. Due to the mirror symmetry, the detuning terms for $x$ and $y$ axis are described in Eqs.(2) by the single function $D(z)$. As follows from the form of Eqs.(2), there is no detuning for chambers invariant over a $90^{\circ}$ rotation; $D(z)=0$ in this case. To give examples, wake functions caused by the wall resistivity are presented below for three simplified cases, namely, for a round chamber, then, for an infinite plane and finally, for a small cylinder. The three cases are sketched at Figs. 1a, $1 \mathrm{~b}$ and $1 \mathrm{c}$.

For the first case of the round vacuum chamber the wake functions can be found in Ref. [2]:

$$
W_{x}(z)=W_{y}(z)=-\frac{2}{\pi b^{3}} \sqrt{\frac{c}{\sigma z}} L, \quad D(z)=0,
$$

where $c$ is a velocity of light, $b$ is the vacuum chamber radius, and $\sigma$ is a wall condactivity.

For the single resistive plane, the trannsverse wake functions follow:

$$
W_{x}(z)=W_{y}(z)=D(z)=-\frac{L}{2 \pi h^{3}} \sqrt{\frac{c}{\sigma z}},
$$

where $h=|\mathbf{h}|$ is the distance from the beam to the plane. This geometry demonstrates the possibility for the detuning wake to be equal to the driving wake.

The final example treats the case of the beam passing along a small resistive cylinder, Fig. (1c); the detuning 
wake is shown to dominate here. Taking into account that the image charge is located at the position $\mathbf{r}^{\prime}=\mathbf{r} \rho^{2} / r^{2}$ and assuming the cylinder radius $\rho$ to be much smaller than the distance between the beam and the cylinder, $\rho \ll r_{0}=$ $|\mathbf{d}|=d$, the longitudinal electric field is:

$$
E_{s}=-\frac{2 \zeta q}{\rho}\left(1+\frac{\rho^{2} \mathbf{r r}_{0}}{r^{2} r_{0}^{2}}\right)+C \ln (r / \rho),
$$

which includes an arbitrary constant $C$. The small dipole term in the brackets reflects a weak dependence of the fields on the source position $\mathbf{r}_{0}$. To find the constant $C$, it can be assumed that this system is bounded by a conducting cylinder with the radius $R \gg r_{0}$. Then the constant $C$ is found by equating the expression for the monopole part of $E_{s}$ to zero at this remote surface, giving $C=\frac{2 \zeta q}{\rho \ln (R / \rho)}$.

Then, one can obtain the integrated transverse force and finally the wakes:

$$
\begin{gathered}
D(z)=-\frac{L}{\pi d^{2} \rho \ln (R / \rho)} \sqrt{\frac{c}{\sigma z}} \\
W_{x}(z)=W_{y}(z)=-\frac{L \rho}{\pi d^{4}} \sqrt{\frac{c}{\sigma z}}
\end{gathered}
$$

Introducing the detuning factors $\kappa_{x}=$ $D(z) / W_{x}(z), \quad \kappa_{y}=D(z) / W_{y}(z)$, the results for the various geometries are expressed as $\kappa_{x}= \pm 1$ for the single plane, $\kappa_{x}= \pm d^{2} /\left(\rho^{2} \ln (R / \rho)\right)$ for the small cylinder, and $\kappa_{x}=0$ for the round chamber.

The driving wake function $W(z)$ for the small cylinder (7) is a factor $\propto \rho / d \ll 1$ smaller than the wake functions of the round chamber (3) or parallel plates (4) with the same aperture. This result demonstrates how the transverse instability can be suppressed by the decrease of the driving wake function. The detuning wakes work in the same direction; they damp the instability even more.

Finally, note that the plane wall result $\kappa_{x}= \pm 1$ is valid not only for the resistive wall wake. It applies as well to the wake generated by a longitudinal variation of the chamber cross-section, when the cross-section is a significantly elongated figure such as a rectangle or ellipse.

\section{COHERENT STABILIZATION BY THE DETUNING WAKE}

The detuning wake modulates the betatron frequencies along the bunch. Such a modulation introduced by means of an RF quadrupole was studied in Ref. [6]. It was shown there that the transverse instabilities can be strongly damped in this case because the particles are kept out of resonance with each other. Following Ref. [6], the numerical results for the influence of the detuning wake on the transverse mode coupling instability are presented below.

Assuming the bunch to consist of particles with the same synchrotron amplitude $a$ and a homogeneous distribution over the synchrotron phase (the so-called air-bag model
[2]), the transverse equation of motion is written

$$
\begin{aligned}
& \frac{d^{2} x(\phi)}{d t^{2}}+\omega_{b}^{2} x(\phi)=F_{x}(\phi) \\
& F_{x}(\phi)=-\frac{N q^{2}}{2 \pi \gamma m L} \int_{-|\phi|}^{|\phi|}\left(W(z) x\left(\phi^{\prime}\right)-D(z) x(\phi)\right) d \phi^{\prime}
\end{aligned}
$$

Here $\phi$ is the synchrotron phase, $\omega_{b}$ and $\omega_{s}$ are the betatron and the synchrotron frequencies, and $N$ is the number of particles in the bunch. An expansion of the deviation $x(\phi)$ over the synchrotron harmonics

$$
x(\phi)=e^{-i \omega_{b} t} \sum_{n=-\infty}^{+\infty} x_{n} e^{-i \alpha \omega_{s} t+i n \phi},
$$

reduces Eq. (8) to a set of algebraic equations for the eigenvector components $x_{n}$ and the eigenvalues $\alpha$ :

$$
\begin{array}{ll}
x_{n}(\alpha-n)=\quad & K \sum_{m=-\infty}^{+\infty} x_{m} K_{n m}, \quad K=\frac{N q^{2}}{2 \pi^{2} \gamma m \omega_{b} \omega_{s} L} \\
K_{n m}=\quad & \int_{0}^{\pi} \cos (n \phi) d \phi \int_{0}^{\phi} W(z) \cos \left(m \phi^{\prime}\right) d \phi^{\prime}- \\
& \int_{0}^{\pi} \cos ((n-m) \phi) d \phi \int_{0}^{\phi} D(z) d \phi^{\prime}
\end{array}
$$

where the influence of the coherent interaction is taken to be small in a comparison with the transverse focusing, $\alpha \omega_{s} \ll$ $\omega_{b}$. To resolve such equations, the sum has to be truncated to a finite number of the modes. In the numerical calculations, five modes were taken initially; then, the results were compared with nine- and fifteen-mode truncations. All the resistive wall wake functions have the following form:

$$
W(z)=-Q / \sqrt{z}, \quad D(z)=-\kappa Q / \sqrt{z},
$$

where $Q$ is the geometry factor. The examples for the detuning factor $\kappa$ are given above.

Figure 2 presents plots for dimensionless eigenvalues $\alpha$ as functions of the dimensionless intensity parameter

$$
\mathcal{I}=K Q / \sqrt{a}
$$

at various detuning factors $\kappa$. The dependence of the mode behavior on this factor is seen to be significant.

The mode coupling instability threshold is least for the symmetric case, $\kappa=0$. At $\kappa=1$, coupling and decoupling thresholds merge (degenerate case) and the beam is stable for any current. This result is valid for any mode truncation, so it appears to be an exact property. A small couplingdecoupling instability area appears again at higher $\kappa$.

Fig. (3) shows the threshold behavior versus coefficient of asymmetry $\kappa$ for the five, nine and fifteen modes calculation. The instability threshold has its minimum for the symmetric chamber, $\kappa=0$. Then it increases with the absolute value of the detuning factor and has two asymmetrical maxima at $\kappa \approx-1.5$ and $\kappa \approx 2$.

The results shown in this figure should be interpreted carefully, taking into account that an asymmetry not only introduces the detuning wake but also changes the driving wake. For instance, the thresholds for the resistive wall, examples a) and b) (Fig. 1) with $h=b$, differ approximately by a factor of $4 \times 1.5=6$, where the factor 4 is related to the driving wake damping and the factor 1.5 is the benefit due to the detuning for $\kappa=-1$, according to the Fig. (3). 

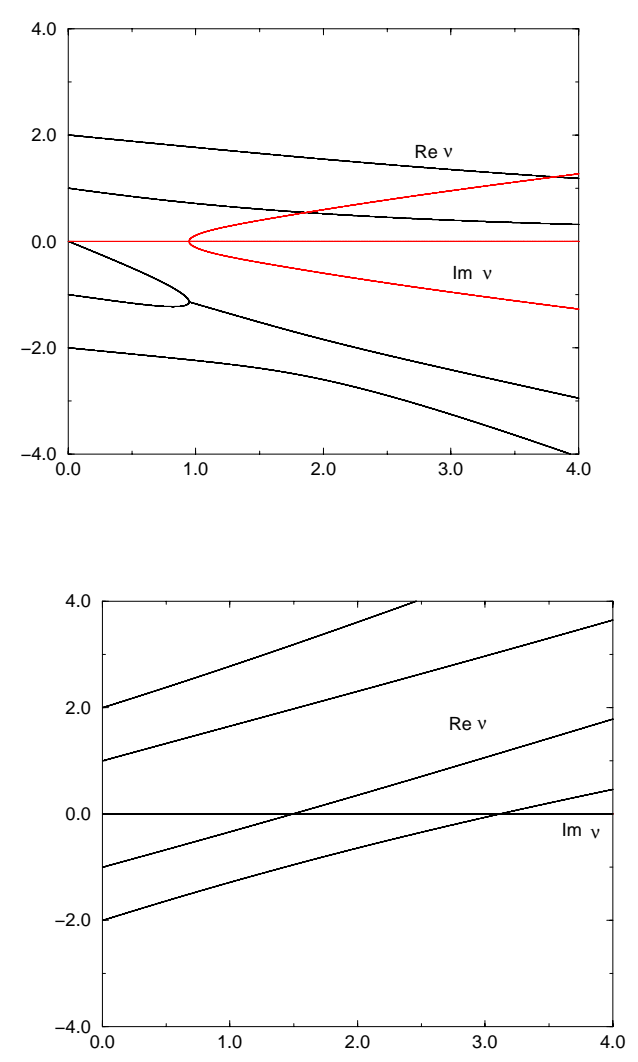

Figure 2: Eigenvalues $\alpha$ versus the intensity parameter $\mathcal{I}$ for various detuning factors $\kappa=0$ (top) and $\kappa=1$ (bottom).

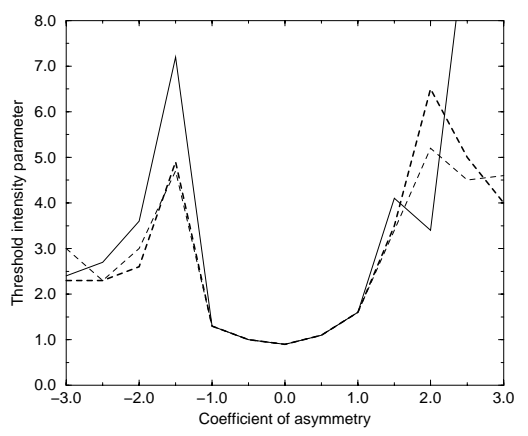

Figure 3: Intensity threshold of the transverse mode coupling instability $\mathcal{I}$ versus the detuning factor $\kappa$ for the 5mode (solid line) 9-mode (thick dash line) and 15-mode (thin dash line) truncations.

\section{CONCLUSIONS}

Only one kind of wake function, called here the driving wake function, has been conventionally taken into account for the beam stability analysis. It has been shown that this conventional approach can lead to significant underestimation of the beam stability thresholds for non-round vacuum chambers. For asymmetric vacuum chamber elements, which are usual in practice, the detuning wake function must be taken into account; conventional codes like MAFIA need to be improved accordingly. For all of the examples here, an asymmetry-driven increase of the detuning wake combines with a decrease of the conventional wake; both of these factors favor beam stability. These properties of asymmetric cross-sections look promising for design of future accelerators.

The authors are grateful to Bruno Zotter, Sam Heifets and Vladimir Shiltsev for interesting discussions. Our special thanks are to Jim Maclachlan and Jeff Holmes for their numerous stylistic corrections of the manuscript.

\section{REFERENCES}

[1] * See also A. Burov and V. Danilov, Phys. Rev. Lett., 82, p. 2286 (1999).

[2] A. Chao, Physics of Collective Beam Instabilities in High Energy Accelerators, John Wiley \& Sons, Inc., 1993.

[3] S. Heifets, A. Wagner, B. Zotter, "Generalized Impedances and Wakes in Asymmetric Structures", SLAC/AP110, 1998

[4] W. Schnell, "Microwave Quadrupoles for Linear Colliders", CLIC Note 34, CERN-LEP-RF/87-24, 1987.

[5] V. Balakin, A. Novokhatsky and V. Smirnov, Proc. 12th Int. Conf. High Energy Accel., (Fermilab, Batavia, 1983), p. 119.

[6] V. Danilov, "On possibility to increase the TMCI threshold by RF quadrupole", Fermilab TM-2033 (1997); Phys. Rev. ST Accel. Beams, 1, 041301 (1998). 